\title{
FINANCIAMIENTO DE LAS MIPYMES DEL SECTOR INDUSTRIAL EN EL MUNICIPIO DE SINCELEJO
}

\section{SMES INDUSTRIAL SECTOR FINANCING IN SINCELEJO}

\author{
Heberto Salas Quintana* \\ Corporación Universitaria Del Caribe, CECAR
}

RECIBIDO: Abril 2 de 2014

ACEPTADO: Junio 28 de 2014

\begin{abstract}
RESUMEN
En este artículo se reportan los resultados de una investigación que tuvo como objetivo analizar las fuentes de financiamiento utilizadas por las micro, pequeñas y medianas empresas (MIPYMES) del sector industrial del municipio de Sincelejo (Colombia) con el fin de caracterizar determinantes claves en su funcionamiento. La muestra fue conformada por 208 empresarios, a quienes se les aplicó un cuestionario tipo likert. La confiabilidad del mismo se hizo con el índice alfa de Cronbach, y se utilizaron tablas de contingencia para procesar la información, así como el coeficiente Chi cuadrado para analizar la asociación entre las variables. Los resultados evidencian que las empresas de este sector utilizan en sus inicios recursos propios para el desarrollo de sus actividades y, luego, al requerir financiamiento, recurren al sector financiero o a la reinversión de sus utilidades. Se concluyó que el acceso al financiamiento sigue siendo un obstáculo para estas empresas y que el acceso a los créditos se les dificulta por causa de los requisitos solicitados como soportes de estos y por las garantías que se les exigen. Además, los recursos que obtienen son utilizados preponderantemente para compra de maquinaria o capital de trabajo.
\end{abstract}

Palabras clave: Fuentes de financiamiento, Mipymes, recursos propios, sector financiero.

Referenciar este artículo:

Salas Quintana, H. (2014). Financiamiento del Sector Industrial de Las Mipymes en el Municipio de Sincelejo, en Desarrollo Gerencial, Revista de la facultad de ciencias económicas, administrativas y contables. Barranquilla (Colombia): Universidad Simón Bolívar. 6 (2). Pp. 207-234.

\footnotetext{
* Contador Público de la Universidad Autónoma del Caribe. Especialista en Finanzas - Convenio Universidad de Cartagena - CECAR. Especialista en Tributación - Universidad Autónoma del Caribe. Candidato a Magíster en Administración de la Universidad Nacional de Colombia. Docente desde 1.987 en distintas universidades de la ciudad de Sincelejo. Correo electrónico: hesaqui@gmail.com 


\begin{abstract}
To analyze financing strategies and establish key aspects related to micro, small and big enterprises in Sincelejo (Colombia) were addressed in this research paper. 208 enterprises were sampled using a Liker questionnaire and its reliability is based on Cronbach's alpha index; to process information was used contingency tables and in order to analyze variable association the coefficient Chi-square test was used as well.

The results show at the beginning that companies use their own financial resources for their business development and when they require funding, these companies resort to the financial sector or reinvest their own profits. As a conclusion, for these companies to access to finance remains an obstacle and bank credits become difficult as well because of the requirements they ask for, such as the required guarantees.
\end{abstract}

On the other side, the resources obtained are predominantly used for purchasing machinery or working capital.

Keywords: Funding sources, SMEs, own resources, financial sector 


\section{INTRODUCCIÓN}

En las diferentes actividades

económicas, la capacidad productiva exigida por el mercado requiere, a su vez, un buen manejo de los recursos financieros. En el caso particular de las Mipymes, es de suma importancia la consecución de recursos de financiamiento que les permitan su sostenibilidad. Por otra parte, esta sostenibilidad empresarial es fundamental en el progreso de un país, pues, su presencia en el mercado es sinónimo de posibilidades de trabajo para la comunidad y esto se refleja en una mejor calidad de vida de sus habitantes.

El censo económico realizado en

Colombia por el DANE en el 2005, recogió información acerca de 1.391 .542 establecimientos empresariales. De estos, el $99,87 \%$, es decir, cerca del total de empresas del país (DANE, 2008) pertenece al sector de las Mipymes. Pero, pese a su representatividad en la economía del país, factores como el acceso a las fuentes de financiación, los tributos, el contrabando, la informalidad y la legislación laboral hacen que muchas de estas empresas desaparezcan antes de dos años de haber sido constituidas. .

La alta incidencia que tienen las Mipymes en la provisión de fuentes de trabajo las convierten en una base fundamental del tejido social, no solo en Colombia sino en la mayoría de países latinoamericanos donde la participación de este sector empresarial impacta en la generación de puestos de trabajo. De manera concreta, según Vera y Mora (2011a, p. 2), en Colombia estas empresas "generan el $30 \%$ de la producción manufacturera, el $43 \%$ del empleo industrial y más del $20 \%$ de las exportaciones no tradicionales"; en tanto que Cohen y Baralla (2012) reportan que su contribución al PIB es del 38,7\%. Este 
porcentaje es bastante significativo, teniendo en cuenta la escasez de fuentes de empleo en el país.

A pesar de su importancia manifiesta, el sector empresarial percibe como un obstáculo su acceso al financiamiento bancario en Colombia. Galán, De Giusti, Mollo y Nóbile (2007) consideran que, en efecto, los pequeños y medianos empresarios identifican la falta de acceso al crédito como el obstáculo más importante que encuentran para desarrollar sus empresas. En un sentido similar, Cohen y Baralla (2012) afirman que, en general, las Mipymes tienen un menor acceso al crédito bancario que las empresas grandes.

Sin embargo, no sólo el deficiente acceso al financiamiento no es el único problema de las Mipymes, sino que, como dice la CMIC (2012), hay una desigualdad en el acceso al crédito que perjudica a las empresas medianas $y$ pequeñas, aspecto este que puede actuar como un inhibidor de su crecimiento. Aún peor es el hecho de que las fuentes de financiamiento y de consecución de recursos a través de las entidades bancarias, tan importantes para este tipo de organizaciones, que por su magnitud no pueden participar de fuentes de financiación más asequibles y menos onerosas, les resta competitividad y disminuye su probabilidad de éxito (Melgarejo, 2007).

En este marco, la presente investigación expresa su plena pertinencia, dado que, en el caso específico del municipio de Sincelejo, no se tiene conocimiento del impacto de los financiamientos recibidos por las empresas locales, como tampoco de su alcance en el entorno. De hecho, en este municipio parece experimentarse un importante rezago, que impide el fortalecimiento económico de este importante sector empresarial. 
No obstante lo anterior, la posición geográfica del municipio es un aspecto que puede favorecer a las Mipymes del sector industrial. La ventaja comparativa que esto le otorga frente a otros municipios del país, en relación con la firma de los Tratados de Libre Comercio, lo puede convertir en un factor de desarrollo y elemento fundamental para el crecimiento económico de la región. A partir del contexto antes descrito y la relevancia de las Mipymes en el desarrollo económico del país, nuestra investigación fue orientada por la siguiente pregunta: ¿Cuáles son las características de las fuentes de financiamiento utilizadas por los empresarios del sector industrial del municipio de Sincelejo?

\section{METODO}

\section{Diseño}

En la investigación se utilizó una metodología mixta, es decir, se combinó lo cualitativo con lo cuantitativo (Pérez Juste, 2006), buscando identificar algunas causas que pueden originar inconvenientes en el acceso de las Mipymes del sector industrial de Sincelejo a los recursos financieros. Por otra parte, se realizó una investigación de corte descriptivo no experimental, esto es, las variables del estudio no fueron alteradas intencionalmente por el investigador (García, González y Ballesteros, 2001), de modo que solo se recogió la información en las condiciones existentes.

\section{Instrumentos}

Para recoger la información, se aplicó una encuesta compuesta por 14 ítems, con preguntas de selección múltiple, algunas dicotómicas y otra politómicas. Al inicio de la encuesta se pide a los empresarios información básica 
para identificar la empresa. Luego, los ítems en el cuestionario se organizaron teniendo en cuenta: 1) las fuentes de financiamiento, 2) los problemas de financiación que se originan en variables o dimensiones internas, y 3) el destino o aplicación por parte de las Mipymes a los recursos financieros provenientes de las fuentes de financiamiento.

La fiabilidad de los ítems se logró aplicando el alfa de Cronbach, coeficiente que produce valores oscilatorios entre $0 \mathrm{y}$ 1. Los valores próximos a 1 indican una alta confiabilidad y los cercanos a cero, confiabilidad baja (Hernández et al, 2006). Este análisis se dividió en tres partes (Tabla 1), considerando: los ítems relacionados con las fuentes de financiamiento para las Mipymes, los relacionados con los problemas de financiación que se originan en variables o dimensiones internas de las Mipymes, y los relacionados con el destino o aplicación por parte de las Mipymes a los recursos financieros provenientes de las fuentes de financiamiento.

Así, en el análisis de la fiabilidad del cuestionario para los constructos relacionados con fuentes de financiamiento, se obtuvo un alfa de Cronbach $=0.901 ;$ para los relacionados con problemas de financiación, el alfa fue de 0,91 y los pertinentes al destino o aplicación de los recursos alcanzaron un valor de 0,9. Además, el cuestionario en su totalidad registró un índice Alfa de Cronbach de 0.891 , lo cual quiere decir que la confiabilidad del instrumento con los 14 ítems es del 86.1\%. Lo anterior indica que el nivel de correlación de las variables en el instrumento es adecuado como, lo que denota a priori una buena consistencia interna (García, González, y Ballesteros, 2001; Hernández et al, 2006). 
Tabla 1. Estadísticos de fiabilidad para el cuestionario piloto y el final

\begin{tabular}{|l|c|c|}
\hline \multicolumn{1}{|c|}{ Constructo } & $\begin{array}{c}\text { Alfa de } \\
\text { Cronbach }\end{array}$ & N de elementos \\
\hline Fuentes de financiamiento para las Mipymes & 0,901 & 5 \\
\hline $\begin{array}{l}\text { Problemas de financiación que se originan en } \\
\text { variables o dimensiones internas de las Mipymes }\end{array}$ & 0,91 & 5 \\
\hline Destino o aplicación de los recursos financieros & 0,90 & 4 \\
\hline Estadísticos de fiabilidad con la 14 variables & 0.891 & 14 \\
\hline
\end{tabular}

Fuente: Encuestas aplicadas, elaboración propia.

Para evaluar la validez de constructo del instrumento, se realizó un análisis factorial exploratorio de las puntuaciones obtenidas. Con el propósito de obtener factores estadísticamente independientes y de máxima variabilidad, la extracción de los mismos se llevó a cabo mediante el método de componentes principales. De esta manera, el primer factor explica un $18,2 \%$ de la varianza, mientras que los siguientes explican entre el $9 \%$ y el $8 \%$, respectivamente, lo que evidencia la importancia relativa del primer factor.
Según Olivo y Batanero (2011), todo esto hace manifiesta la validez del constructo, dado que el porcentaje de varianza del primer factor es claramente superior al explicado por los restantes. Además, los factores restantes explican cada uno aproximadamente la misma varianza. Antes de continuar, cabe decir que todos los procedimientos estadísticos se realizaron con el programa SPSS Statistics 20 . 


\section{Procedimiento}

El proceso investigativo se realizó en tres etapas: 1) revisión de fuentes $y$ construcción de la propuesta e instrumentos; 2) recolección de la información; y 3) análisis y comunicación de resultados. En la primera etapa se hizo un análisis del estado de la cuestión, fundamentando los elementos necesarios para hacer el análisis de la información obtenida. Atendiendo los referentes conceptuales y de investigación, luego se ajustó la propuesta inicial y se elaboró el cuestionario. En la segunda etapa se contactaron a los representantes legales de las empresas y se aplicaron los cuestionarios. Para el análisis de los resultados (etapa 3), luego de recoger la información, se procedió a construir la base de datos en SPSS, en la cual se crearon las variables tipo de empresa, año de constitución, forma legal de constitución y, por último, las correspondientes a cada objetivo. Los procedimientos estadísticos que se realizaron fueron los siguientes: para el análisis de fiabilidad del instrumento se utilizó el coeficiente Alfa de Cronbach. También se hizo un análisis descriptivo a los ítems de identificación de la empresa y posteriormente se practicó el procesamiento de la información, utilizando tablas de contingencia y el estadístico chi-cuadrado de Pearson para analizar la asociación de cada una de las variables con el tipo de empresa y su forma legal de constitución. De esta forma, se buscó ofrecer una mayor comprensión del fenómeno estudiado (Hernández, Fernández y Baptista, 2006).

\section{Participantes}

La población objeto de este estudio estuvo conformada por un total de 502 empresas. De estas, 60 fueron constituidas a través de personas jurídicas y 442 por medio de personas naturales. 
Esta población se constituyó a la vez en la muestra invitada (Ospina, 2001), a la cual se decidió aplicar la encuesta. Como criterio de inclusión, se consideró a las Mipymes que habían renovado la matrícula mercantil a partir del año 2011, información encontrada en una base de datos proporcionada por la Cámara de Comercio de Sincelejo, la cual se utilizó como marco muestral. La muestra aportante fue de 208 empresas, y su distribución se puede apreciar en la Tabla

2. La reducción se debe a que algunas direcciones estaban erradas y a que algunos empresarios se negaron a responder la encuesta o no se encontraban en el momento de la aplicación.

Tabla 2. Distribución de muestras según el tamaño de la empresa y su forma legal de constitución

\begin{tabular}{|c|c|c|c|}
\hline \multicolumn{4}{|c|}{$\begin{array}{c}\text { DISTRIBUCION DE MUESTRAS SEGÚN EL TAMAÑO } \\
\text { DE LA EMPRESA Y SU FORMA LEGAL DE } \\
\text { CONSTITUCIÓN }\end{array}$} \\
\hline $\begin{array}{c}\text { TIPO DE } \\
\text { EMPRESA }\end{array}$ & $\begin{array}{l}\text { PERSONA } \\
\text { NATURAL }\end{array}$ & $\begin{array}{l}\text { PERSONA } \\
\text { JURIDICA }\end{array}$ & TOTAL \\
\hline MICROEMPRESAS & 156 & 40 & 196 \\
\hline $\begin{array}{l}\text { PEQUEÑAS } \\
\text { EMPRESAS }\end{array}$ & 2 & 6 & 8 \\
\hline $\begin{array}{l}\text { MEDIANAS } \\
\text { EMPRESAS }\end{array}$ & 1 & 3 & 4 \\
\hline TOTAL & 159 & 49 & 208 \\
\hline
\end{tabular}

Fuente: Elaboración propia del autor a partir de información suministrada por la Cámara de comercio de Sincelejo (2014).

\section{Conceptos básicos}

Las Mipymes aportan innovación y promueven la eficiencia en los sistemas productivos, pero a nivel internacional no hay consenso sobre su definición. Los países establecen parámetros de categorización de acuerdo con sus propias particularidades contextuales, que son diferentes de un país a otro. En Colombia, el artículo 43 de la Ley 1450 del año 2011 (Plan Nacional de desarrollo, 2011, p. 928) establece que la "empresa es toda unidad de explotación económica realizada por persona natural o jurídica en actividades empresariales, agropecuarias, industriales, comerciales o de servicios". Conforme a la misma ley, se clasifican de la siguiente manera: 
Tabla 3. Clasificación de las Mipymes según el tipo de empresas en Colombia

\begin{tabular}{|l|l|l|}
\hline $\begin{array}{l}\text { TIPO DE } \\
\text { EMPRESA }\end{array}$ & $\begin{array}{l}\text { PLANTA } \\
\text { DE } \\
\text { PERSONA } \\
\text { L }\end{array}$ & $\begin{array}{l}\text { ACTIVOS } \\
\text { TOTALES } \\
\text { EN SMMLV }\end{array}$ \\
\hline $\begin{array}{l}\text { MICROEM } \\
\text { PRESA }\end{array}$ & HASTA 10 & $\begin{array}{l}\text { MENOR A } \\
500\end{array}$ \\
\hline $\begin{array}{l}\text { PEQUENA } \\
\text { S } \\
\text { EMPRESA } \\
\text { S }\end{array}$ & 11 A 50 & 501 A 5.000 \\
\hline $\begin{array}{l}\text { MEDIANA } \\
\text { S } \\
\text { EMPRESA } \\
\text { S }\end{array}$ & 51 A 200 & $\begin{array}{l}5.001 \mathrm{~A} \\
15.000\end{array}$ \\
\hline
\end{tabular}

SMMLV para el año

2014: $\$ 616.000$

Fuente: Ley 1.450 año 2011 Congreso de la

República de Colombia, elaboración propia.

Otro concepto de interés en este artículo es el de industria, el cual es considerado por el DANE (2008) como la transformación física y/o química de materiales y componentes en productos nuevos, ya sea que el trabajo se efectúe con máquinas o a mano, en una fábrica o a domicilio, o que los productos se vendan al por mayor o al por menor.
Es clara la importancia y dinamismo de las Mipymes en la economía de cualquier país en dimensiones como número de establecimientos, solución al desempleo, producción, distribución equitativa de recursos, entre otros indicadores. De ahí el interés creciente por investigar sus necesidades de superar debilidades $\mathrm{y}$ fortalecer su permanencia en sus respectivos asentamientos (Vera y Mora, 2011b). Estas unidades productivas, además de producir bienes y servicios, generan empleos e ingresos para los distintos grupos sociales, y desarrollo económico en los lugares donde desarrollan sus actividades.

Otro concepto importante en nuestro trabajo es el de recurso, que se define como todo movimiento que afecte positivamente el capital de trabajo de los negocios, es decir, a la diferencia resultante de deducir al activo circulante el pasivo de la misma naturaleza. Entre

\footnotetext{
* Salario Mínimo Legal Vigente.
} 
los recursos que maneja una empresa, el efectivo es de vital importancia, y este se puede calcular, determinar u obtener a través de un estado financiero denominado Estado de Flujo de Efectivo, que, según Amador, Romano y Cervera (2007), es un estado que proporciona información sobre los orígenes y el uso que se da a las entradas de efectivo y otros activos líquidos equivalentes, clasifica los movimientos por actividades e indica la variación neta de dicha magnitud en el ejercicio.

Esta herramienta facilita a los empresarios información para saber de dónde provino el efectivo y como se aplicó en un determinado periodo, facilitando su manejo adecuado y una toma de decisiones adecuada para reorientar la empresa. Su importancia radica en el interés que ha de tener toda empresa por permanecer informada sobre sus finanzas, lo que está estrechamente relacionado a su pasado, presente $\mathrm{y}$ futuro.

Por último, se entiende como acceso al financiamiento la posibilidad que tiene un ente económico de adquirir recursos para realizar cualquier actividad de su interés (Castaño y Arias, 2013), es decir, la opción de que su solicitud de endeudamiento para conseguir recursos financieros sea aprobada oportunamente. En algunos casos, quienes adquieren este tipo de obligaciones son agentes económicos cuyo acceso al financiamiento no es más que la reinversión de sus utilidades, o la consecución de medios financieros para realizar operaciones de corto plazo (Vera y Mora, 2011a) que les permitan satisfacer sus necesidades y prevalecer en su entorno.

De lo planteado por Vera y Mora, se puede inferir la importancia del acceso al financiamiento y a los productos del mercado financiero en el ejercicio de las 
actividades empresariales, ya que dicho acceso "facilita la puesta en marcha de pequeñas firmas, el crecimiento $\mathrm{y}$ aprovechamiento de oportunidades de inversión, el desarrollo e innovación en nuevos productos, así como la elección de estructuras organizativas más eficientes y carteras de activos más adecuadas" (TheWorld Bank, 2008, citado porVera y Mora, 2011a, p.3).

\section{RESULTADOS}

Según su tamaño, las empresas mostraron una tendencia a acudir a los mismos tipos de fuentes $\left(\chi^{2}=34,778\right.$; $\mathrm{P}<0,05)$. Monge y Rodríguez (2011) proponen diversas fuentes de financiamiento que podrían utilizar las empresas para financiarse, de acuerdo con su grado de madurez y riesgo, a saber: 1) capital semilla, 2) inversionistas ángeles, 3) financiamiento bancario y 4) fondos públicos no reembolsables, los cuales se han utilizado en muchos países para mejorar la capacidad de innovación y productividad de estas empresas.

En cuanto a las variables que más dificultan el acceso a los créditos, el $63,46 \%$ de los empresarios encuestados destaca los requisitos que se les piden como soportes de estos, y 11,53\% ve como dificultad las garantías exigidas para otorgárselos. El $25 \%$ restante menciona otras dificultades, así: los costos que les generan tales créditos $(9,61 \%), y$ los que consideran más de una o ninguna $(15,38 \%)$.

Además de lo anterior, hay acuerdo intragrupos, es decir, según la forma de constitución de las empresas $\left(\chi^{2}=19,730\right.$; $\mathrm{P}<0,05)$ y según el tipo de empresas $\left(\chi^{2}=\right.$ 35,760; $\mathrm{P}<0,05)$, con relación al tipo de variables que dificultan el crédito. De modo que las dificultades de una determinada clase tienden a afectar a las 
más pequeñas, e igualmente, las empresas son afectadas según su forma de constitución.

En este último sentido, los empresarios consideran que se les exige más requisitos y garantías a las empresas constituidas como personas naturales que a las que aparecen como personas jurídicas. Y este panorama se agrava si se tiene en cuenta que las restricciones al crédito y la falta de garantías son los principales obstáculos para el desarrollo de este sector empresarial (Rodríguez y Zorrilla, 2007), y que las entidades financieras someten a los empresarios a unos análisis de riesgos extremadamente rigurosos, que, en ocasiones, se convierten en filtros infranqueables para estos (Pérez y Jiménez, 2004)..

En relación a lo anterior, "se puede afirmar que las fuentes de financiamiento para el microcrédito, son similares a las utilizadas para cualquier modalidad de crédito en Colombia, salvo los volúmenes y los procesos que se siguen para su otorgamiento" (Serrano, 2009, p. 21). Y en términos generales, se considera el acceso a las fuentes de financiación como fundamental para el fortalecimiento del dinamismo empresarial, ya que propicia la generación, crecimiento y desarrollo de estas empresas.

Para los empresarios, la financiación es importante para la sostenibilidad de sus negocios; no obstante, $38,46 \%$ de ellos considera que les representa unos costos altos, 28,84\% los considera medios, 15,38\%, bajos, y el $17,3 \%$ considera que la consecución de un crédito no les representa ningún costo significativo. Además, se presenta una asociación altamente significativa $\left(\chi^{2}=\right.$ $17,245 ; \mathrm{P}<0,05)$ entre los costos de la financiación y el tipo de empresa. Es decir, los costos generados por un crédito pueden considerarse un mayor o menor 
obstáculo en relación al tamaño de la empresa.

En atención a lo anteriormente expuesto, se destaca el reto que tienen los países en crear condiciones que favorezcan el desarrollo de este sector empresarial, es decir, "poder focalizar las acciones de fomento y crear una institucionalidad de apoyo para mejorar las condiciones de financiamiento (...) la simplificación de trámites, la capacitación, la creación de agencias sectoriales y el establecimiento de regulaciones que buscan diferenciar a estas empresas de las grandes" (Corcuera, Carrasco y Cárdena, 2010, p.11); por lo que la falta de políticas dirigidas a que las Mipymes tengan acceso a fuentes de financiación oportuna y ventajosa inhiben su crecimiento sostenido(ONU, 2006).

En cuanto a la importancia concedida por los empresarios a la capacitación en administración de negocios de su personal clave para el desarrollo y sostenibilidad de las Mipymes, el 57,7\% le da un alto valor, el $25 \%$, medio, el $9,61 \%$, baja, y un $7,7 \%$ no le concede ninguna importancia. Además, hay consenso entre los empresarios en que este tipo de capacitaciones es necesario para la sostenibilidad de sus negocios. Esto contradice lo reportado por Merino y Díaz (2008), quienes consideran que los empresarios capacitan muy poco en cuestiones de negocio a su personal clave, lo que, en consecuencia, incide en la insatisfacción de estos con sus trabajos y, por ende, en el desarrollo de las empresas.

Para los empresarios encuestados, las variables con mayor incidencia en el desarrollo y sostenibilidad de las Mipymes son las fuentes de financiación a costos razonables $(63,46 \%)$, capacitación en administración de negocios de sus talentos claves $(19,23 \%)$, la normatividad existente $(11,53 \%)$ o una 
combinación de ellas $(5,76 \%)$. Además, estas respuestas resultaron homogéneas intra-grupos, tanto en relación al tipo de empresas $\left(\chi^{2}=33,316, \mathrm{P}<0,05\right)$ como en la forma legal de constituirlas $\left(\chi^{2}=9,764\right.$, $\mathrm{P}<0,05)$. Esto parece contraproducente para el desarrollo empresarial, ya que, según García, Barona y Madrid (2013), una deuda puede ser beneficiosa, debido a que la financiación mediante esta fuente es menos costosa que por otra vía, disminuyendo gastos financieros.

Los recursos que las Mipymes de Sincelejo obtienen de las diferentes fuentes de financiación son aplicados de la siguiente manera: compra de maquinaria $(40,38 \%)$, capital de trabajo $(19,23 \%)$, compra de inmuebles $(3,84 \%)$, usos varios $(34,61 \%)$ u otros usos no especificados (1,92\%). Díaz y GarcíaHerreros (2009), así como de Rodríguez (2003) manifiestan, con base en datos de Fundes, que el $46 \%$ del dinero proveniente del financiamiento de las Mipymes se destina a la inversión en capital de trabajo, como: mano de obra, materia prima, insumos o reposición de activos físico entre otros; mientras que el $24 \%$ lo invierte en la compra de maquinaria y equipo. Las dos aplicaciones son indispensables para la operación, desarrollo de actividades industriales y expansión de cualquier empresa manufacturera que quiera permanecer en el mercado.

En la Tabla 4 se presentan algunos resultados que informan sobre el conocimiento de los empresarios en torno al sector financiero. En esta, los P-values menores que 0,05 indican los acuerdos intra-grupos; por ejemplo, existe relación altamente significativa entre grupos por tamaño de empresas y según la forma legal de constituirla en cuanto a la mayor facilidad que poseen de acceder a un crédito en el sector financiero que con particulares $\left(\chi^{2}=12,445, \mathrm{P}<0,05\right) \quad\left(\chi^{2}=\right.$ 
$3,925, \mathrm{P}<0,05)$. Hay otros dos aspectos en

los que se presentan acuerdos altamente

significativos por tamaño de empresa:

costos más bajos en créditos del sector

financiero que con particulares $\left(\chi^{2}=\right.$

$17,560, \quad \mathrm{P}<0,05)$ y conocimiento de

instrumentos financieros $\quad\left(\chi^{2}=10,83\right.$,

$\mathrm{P}<0,05$ ). En la Tabla 4, las sumas

horizontales por tipo de persona y por

tamaño de empresas registran el tamaño

de la muestra en cada una de las entradas.

Tabla 4. Conocimiento del sector financiero, con su chi-cuadrado de Pearson correspondiente

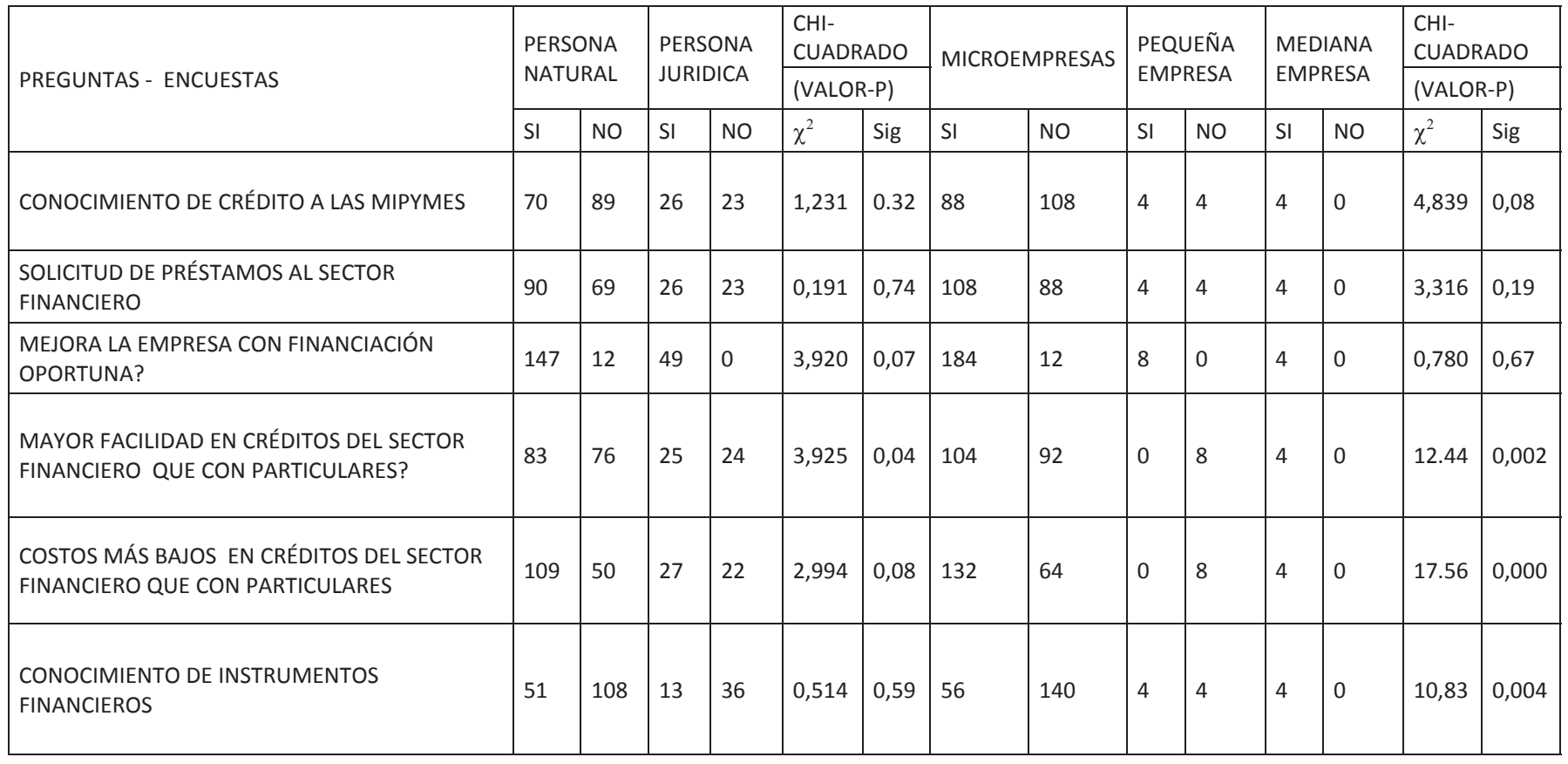

Fuente: Encuestas con cálculos de elaboración propia. 
Otros resultados de la Tabla 4 indican que el 55,76\% de los empresarios encuestados ha solicitado un préstamo al sector financiero. $\mathrm{Y}$ en cuanto al conocimiento de la existencia de créditos para las Mipymes y sus potenciales beneficios para estas, 94,23\% de los empresarios reconoce que una financiación oportuna mejoraría su empresa y $65,38 \%$, que se pueden conseguir costos más bajos con el sector financiero que con particulares. Pero $69,23 \%$ de ellos dice desconocer instrumentos financieros. Además, como puede verse, la cantidad de empresarios que conoce la existencia de créditos para las Mipymes es muy similar a la de los que dicen desconocerlo.

Algo similar sucede en lo referido al hecho de reconocer una mayor facilidad de acceso a un crédito con el sector financiero que con particulares, cuestión que contradice lo planteado por la CMIC (2012) cuando afirma que, en
Colombia, el acceso al financiamiento bancario es visto por el sector empresarial como un obstáculo. Sin embargo, esta respuesta parece indicar cierta incongruencia entre los encuestados, ya que si sólo el 23,07\% (48 empresas) ha accedido a créditos del sector financiero, ¿cómo van a considerar el 51,92\% (108empresas) que hay mayor facilidad de acceso al crédito en el sector financiero que con particulares?, es decir, parecería que sin vivir la experiencia de un crédito en el sector financiero, han dado una respuesta positiva respecto al acceso a este.

Finalmente, al analizar las tasas de interés para libre inversión o microcrédito, se halla que normalmente el sector financiero los otorga a límites bastante cercanos al tope fijado para la tasa de interés de usura. Uno de los principales factores que incide en el costo del dinero es el fenómeno de la inflación, 
pero en la Tabla 5 se observa que mientras el índice de inflación tiende a la baja, la tasa de interés tiende al alza.

Para la década de los noventa, en efecto, la relación tasa de interés-índice de inflación osciló entre 1,59 y 3,29. Pero, en los últimos 7 años (2.007-2.013), esta relación para el caso de la tasa de libre inversión ha oscilado entre 5,60 y 16,11 y para la tasa de microcrédito, entre 5,96 y 27,55 . Estas relaciones confirman lo costoso que le resulta a las Mipymes obtener financiación con el sector financiero cuando logran obtener un crédito. Todo esto con la complacencia y visto bueno del Gobierno Nacional, que es el encargado de fijar las tasas de interés, y normalmente lo hace con base en la información que le suministra el oligopolio (cuasi monopolio) del sector financiero colombiano. 
Tabla 5. Comparativo tasas de interés de usura para los créditos de consumo, ordinario, y microcrédito versus índices de inflación

\begin{tabular}{|c|c|c|c|c|c|c|c|c|c|c|c|c|c|c|}
\hline \multirow{2}{*}{ CONCEPTO } & \multirow{2}{*}{$\begin{array}{c}\text { UNI } \\
\text { D }\end{array}$} & \multicolumn{13}{|c|}{ AÑOS } \\
\hline & & 1990 & 1991 & 1992 & 1993 & 1994 & 1995 & 2007 & 2008 & 2009 & 2010 & 2011 & 2012 & 2013 \\
\hline $\begin{array}{ll}\text { Interés bancario } \\
\text { corriente }\end{array}$ & $\%$ & 34.3 & 36.4 & 32.2 & 35.9 & 38.8 & 42.7 & 21.3 & 21 & 17.3 & 14.2 & 19.4 & 20.9 & 19.9 \\
\hline $\begin{array}{l}\text { Techo tasa interés } \\
\text { usura ( crédito de } \\
\text { consumo } \\
\text { ordinaria) }\end{array}$ & $\%$ & 51.41 & 54.62 & 48.23 & 53.81 & 58.14 & 64.08 & 31.89 & 31.53 & 25.92 & 21.32 & 29.09 & 31.34 & 31.25 \\
\hline $\begin{array}{l}\text { Techo tasa interés } \\
\text { usura } \\
\text { (microcrédito) }\end{array}$ & & & & & & & & 33.93 & 33.93 & 33.93 & 36.89 & 50.18 & 53.45 & 53.45 \\
\hline Índice de inflación & $\%$ & 32.36 & 26.82 & 25.13 & 22.60 & 22.59 & 19.46 & 5.69 & 7.67 & 2.00 & 3.17 & 3.73 & 2.44 & 1.94 \\
\hline 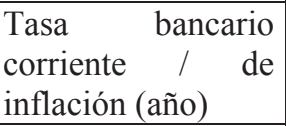 & $\begin{array}{c}\text { Vece } \\
\text { s }\end{array}$ & 06 & 1.36 & 1.28 & 1.59 & 1.72 & 2.20 & 3.74 & 2.74 & 8.64 & 4.48 & 5.20 & 8.56 & 10.23 \\
\hline $\begin{array}{l}\text { Tasa consumo - } \\
\text { ordinaria / índice } \\
\text { de inflación (año) }\end{array}$ & $\begin{array}{l}\text { Vece } \\
\text { s }\end{array}$ & 1.59 & 2.04 & 1.92 & 2.38 & 2.57 & 3.29 & 5.60 & 4.11 & 12.96 & 6.73 & 7.80 & 12.84 & 16.11 \\
\hline $\begin{array}{l}\text { Tasa microcrédito } \\
\text { /índice de inflación } \\
\text { (año) }\end{array}$ & $\begin{array}{c}\text { Vece } \\
\mathrm{s}\end{array}$ & & & & & & & 5.96 & 4.42 & 16.97 & 11.64 & 13.45 & 21.91 & 27.55 \\
\hline
\end{tabular}

Fuente: Súper Financiera - DANE. Elaboración propia

\section{DISCUSIÓN}

Para efectos legales, de las 208 Mipymes

que integran la muestra efectiva, 159 se encuentran constituidas por personas

naturales, y 49 como personas jurídicas, que, a su vez, se encuentran organizadas bajo las siguientes formas de sociedad:

limitadas (7), en comandita simple (4), por acción simplificada (26), colectiva (3) y anónima (9). Al analizarse la antigüedad de las Mipymes de este sector económico, se observa que la gran mayoría $(98,07 \%)$ opera desde hace 15 años o menos en la ciudad de Sincelejo.

En lo que concierne al tipo de financiación con que contaron al iniciar el negocio, $61,53 \%$ inició con recursos propios, $11,53 \%$ con recursos familiares, $5,76 \%$ con recursos de particulares, 
$11,53 \%$ con recursos del sector plazo, poco uso de los programas de financiero, $7,69 \%$ con otro tipo de fomento para el sector, desconocimiento financiación y $1,9 \%$ con alguna de los posibles mercados que se deben combinación de los anteriores. Cabe atender, tanto nacionales como destacar al respecto que de las empresas constituidas como persona natural, $67,3 \%$ inició sus operaciones con recursos propios y que el $42,85 \%$ de las empresas constituidas bajo la figura de persona jurídica también inició sus operaciones con este tipo de recursos. Así, en general, el $61,53 \%$ de las empresas de la muestra inició sus operaciones con este tipo de recursos, lo que refuerza lo reportado por Monge y Rodríguez (2010), quienes afirman que en su mayoría las empresas se financian con recursos internos y su acceso al financiamiento externo es restringido.

Para Beltrán (2010), además del poco capital semilla, otras causas por las que las Mipymes no son competitivas en Colombia son: altos niveles de endeudamiento concentrado en el corto 
trabajos consultados (Castelló, 1994; necesidades, puede achacarse al Fitzgerald, 2007; Valero, 2010) atribuyen desconocimiento, la falta de información la causa de este problema a factores externos a la empresa, y proponen como solución que se creen nuevos mecanismos de apoyo financiero, procedentes del sector público o del privado.

En este mismo sentido, otras propuestas basan su explicación en la gestión financiera de sus propietarios o administradores, planteando la necesidad de fortalecer programas que los capaciten (Vera y Mora, 2011a). Ambos grupos de estudio son complementarios, si se considera que las explicaciones aportadas por los diferentes autores contribuyen a una mayor comprensión de los problemas de las Mipymes, quedando en evidencia que el uso exclusivo de recursos propios en este sector empresarial puede ser un impedimento para el desarrollo y crecimiento de las mismas; pero también, otro impedimento para acceder a $(13,46 \%)$ y otras fuentes $(5,76 \%)$.

programas de apoyo que se adecuen a sus 
De acuerdo con los resultados anteriormente descritos, se puede concluir que: las empresas del sector industrial del municipio de Sincelejo inician mayoritariamente sus operaciones con recursos propios, familiares o de particulares. Muy pocas iniciaron operaciones con recursos de otros sectores (financiero, apoyo del Gobierno Nacional). Especialmente las constituidas como persona natural, inician sus operaciones con recursos propios y entre las fuentes de financiamiento a las que han tenido acceso estas empresas, se destacan los prestamistas informales.

Con el negocio en marcha y ante la necesidad inminente de un crédito, las fuentes de financiamiento a las que más recurren estas Mipymes son los recursos del sector financiero, los recursos propios y la reinversión de sus utilidades. Por otro lado, existe la tendencia, por tamaño de empresa, a acudir a las mismas fuentes de financiamiento. Además, hay acuerdo intra-grupos por tamaño de empresa en que hay mayor facilidad de acceso a créditos del sector financiero que con particulares, y también hay un consenso casi unánime entre los empresarios del sector industrial del municipio de Sincelejo en que una financiación oportuna mejoraría su empresa, y en que es posible conseguir costos más bajos en el sector financiero que con particulares.

Las variables que más dificultan el acceso a los créditos de las Mipymes del sector industrial del municipio de Sincelejo son los requisitos que se les piden como soportes y las garantías que se les exigen para otorgárselos. Asimismo, hay acuerdo intra-grupos según la forma de constitución de las empresas y según el tamaño de las mismas, en que las dificultades de un determinado tipo tienden a afectar más a las microempresas que a las pequeñas y medianas. 
Otra dificultad que tienen las Mipymes de este sector para la financiación de sus negocios está asociada con los altos costos que les representa. Los costos de financiación son considerados un obstáculo, dependiendo del tamaño de la empresa. Y los recursos que obtienen estas Mipymes son utilizados preponderantemente para compra de maquinaria y capital de trabajo.

\section{REFERENCIAS}

Amador, S. Romano, J. y Cervera, M. (2007). El estado de flujos de efectivo. Recuperado en http://www.contabilidad.tk/elestado-de-flujos-de-efectivo.html
Esto puede causar cierto rezago en las empresas del sector industrial del municipio de Sincelejo, que se agrava por el hecho de que ellas no participan en programas de apoyo adecuados a sus necesidades, quizás por desconocimiento de estos, falta de información adecuada o por las limitaciones que tienen para acceder a ellos.
Cámara de comercio Sincelejo (2.014). Mipymes sector industrial del Municipio de Sincelejo, inscritas en el Registro mercantil. Medio electrónico.

Castaño, C. y Arias, J. (2013). Análisis

Beltrán, A. (2010). Los 20 problemas de la pequeña y mediana empresa, en financiero integral de empresas colombianas 2009-2010: Colombia. RevistaSotavento, 7(7), perspectivas de competitividad $8-15$. 
regional. Revista Entramado, 9(1), 84-100.

Castelló, E. (1994). Los planes de apoyo del sistema financiero a las pymes. Revista Perspectivas del sistema financiero, (48), 246-254.

Cohen, M. y Baralla, G. (2012).La situación de las PyMEs en América Latina. Recuperado enhttp://www.ieral.org/images_db /noticias_archivos/2157La $\% 20$ situaci $\%$ C3\%B3n\%20de\% 20las\%20PyMEs\%20en $\% 20 \mathrm{Am} \%$ C3\%A9rica\%20Latina.pdf

CMIC. (2012).Financiamiento y fortalecimiento de mipymes. Recuperado de: http://www.cmic.org/agendaindust ria/AGENDA\%20FINANCIAMI ENTO.pdf
Corcuera, G. Carrasco, R. y Cárdenas, C. (2010). Marcos legales para el fomento a la MIPYME en América Latina. Análisis comparado para Colombia, Chile, Perú y Venezuela. Serie documentos de trabajo $n^{\circ} 3$. Fundes. Recuperado en http://www.fundes.org/uploaded/co ntent/publicacione/2136344480.pdf

DANE (2008). Síntesis del proceso de cierre del Censo General 2005. Recuperado en www.dane.gov.co/censo/files/sinte siscorregido.pdf

Días, A. \& Garcia herreros, L. (2009). Caracterización de la pyme en la industria manufacturera de la ciudad de Cartagena. Subsector D22: Actividades de edición e impresión y de reproducción de 
grabaciones. Cartagena:

Universidad de Cartagena.

FitzGerald, V. (2007). Desarrollo financiero y crecimiento económico: una visión crítica. Revista Principios: estudios de economía política, (7), 5-30.

Galán, L. De Giusti, E. Mollo, G. y Nóbile, C. (2007). La problemática del sector pyme región este de la provincia de Buenos Aires. Recuperado en http://www.econo.unlp.edu.ar/uplo ads/docs/doc_trab_8.pdf

García, J. González, M. y Ballesteros, B. (2001). Introducción a la investigación en educación. Madrid: Editorial Uned. innovación en las Mipyme iberoamericanas.Revista Estudios gerenciales, 29(126), 12-16.
Hernández, R., Fernández, C. \& Baptista, P. (2006).Metodología de la investigación. 4ta. edición. México, McGraw-Hill.

Melgarejo, (2007). Desempeño económico: diferencias de pequeñas empresas clasificadas según la estructura de la propiedad del capital. Revista de estudios cooperativos, (93), 7-38.

Merino, M. y Díaz, A. (2008). El estudio del nivel de satisfacción laboral en las empresas públicas y privadas de Lambayeque. Revista Estudios empresariales, 1(1), 132-142.

García, D. Barona, B. \& Madrid, A. Monge, R. \& Rodríguez, J. (2010). (2013). Financiación de la Financiamiento a la inversión de 
las pymes en Costa Rica.

Recuperado

de:

enwww.eclac.cl/publicaciones/xm

1/1/41501/1c13261pe.pdf

Monge, R. \& Rodríguez, J. (2011).

Caracterización de las PYMES en

Costa Rica mediante el empleo del

Análisis de Conglomerados o

CLUSTER. Revista Tec

Empresarial, 5(1), 8-19.

Moreno, P. Hernández, G. Mendoza, F. Vargas, T. y Villegas, E. (2009). Caracterización de las fuentes de financiamiento para las MiPyMEs en el Estado de Hidalgo. Recuperado en www.uaeh.edu.mx/investigacion/ice a/LI_SisInfoFin/Herib_More/pymeshi dalgo.pdf. ambientales: identificación de oportunidades, políticas e instrumentos Estudios de caso de: Argentina, Chile, Colombia y México. Recuperado en www.eclac.org/publicaciones/xml/6/ 26826/LCW42.pdf

Olivo, E. \& Batanero, C. (2011). Análisis de validez de constructo de un instrumento de medición utilizando análisis factorial. Recuperado en repensarlasmatematicas.files.word press.com/2012/03/olivobatanero-2011.pdf

Ospina, D. (2001). Introducción al muestreo. Bogotá: Universidad Nacional de Colombia.

Pérez, C. y Jiménez, E. (2004). Análisis

Naciones Unidas (2006). Las pymes en el del riesgo de crédito en las mercado de bienes y servicios Sociedades de Garantía Recíproca. 
Revista Estrategia financiera, (207), 28-35.

Pérez Juste, R. (2006). Evaluación de programas educativos. Madrid: Editorial La muralla S.A.

Plan Nacional de desarrollo (2011). Ley 1450 de junio de 2011. Recuperado en www.epssura.com/index.php.

Rodríguez, A. (2003). La realidad de la pyme colombiana desafío para el desarrollo. Recuperado en www.fundes.org/uploaded/content/ publicacione/1241969270.pdf

Rodríguez, M. y Zorrilla, J. (2007).Las restricciones financieras como obstáculo al desarrollo de las pymeslos casos de España, Portugal y México. Recuperado en dialnet.unirioja.es/servlet/articulo? codigo $=2232628$

Romero, F. (2013). Variables financieras determinantes del fracaso empresarial para la pequeña y mediana empresa en Colombia: análisis bajo modelo Logit Revista Pensamiento \& Gestión(34), 235-277.

Serrano, J. (2009). Micro finanzas e instituciones micro financieras en Colombia. Recuperado enwww.eclac.cl/publicaciones/xm 1/2/36802/1c13055p.pdf

Valero, M. (2010). El apoyo financiero oficial a la internacionalización en la actual crisis económica. Revista de Economía, (583), 57-63.

Vera, M. \&Mora, E. (2011a).Indicadores contables y acceso al 


\begin{tabular}{|c|c|}
\hline financiamiento en pymes & Vera, M. \& Mora, E. (2011b). Líneas de \\
\hline colombianas. & investigación en micro, pequeñas \\
\hline www.unicafam.edu.co/EventoAca & y medianas empresas. Revisión \\
\hline demico_1/flash/Articulos/a17.pdf & y desarrollo \\
\hline & Colombia. \\
\hline & $213-22$ \\
\hline
\end{tabular}

